liver and dropsy, from spirit drinking; he was many months ill, but ultimately recovered. When last seen, they were in robust health, but had bloated countenances.

2nd.-A family consisting of three sons and three daughters. They all had the characters of the scrofular diathesis strongly marked - fair hair, clear complexion, blue eyes, thick lips, \&c. The three daughters died of phthisis before the age of thirty; one son of tubercles of the brain-the two remaining sons are still alive, of irregular habits, but in apparently good health.

3rd.-A young man at the age of eighteen showed every symptom of approaching tuberculosis; he had already lost one brother and a sister by phthisis. His medical man advised him to relinquish a profession of a sedentary nature, in consequence of the abore facts. He did so, but unfortunately became dissipated. He is now alive, about forty years of age, a confirmed drunkard, and has suffered several times from delirium tremens.

We gather, therefore, from the foregoing observations, that tubercular deposits are eminently dependent, as the primary if not the sole cause on a diminution of the vital force.-2ndly. That in phthisis the body is rapidly consumed by the combination of its elements with oxygen.-3rdly. That the consumption of the body may be retarded hy the ingestion of certain highly carbonized substances, as cod-liver oil, \&c.-4thly. That it is probable the rapid oxidation of the body may be checked, or entirely prevented, by the use of alcohol, or of some agent acting in the same way.

November, 1852.

\section{A CASE OF PELVIC DISTORTION, IN WHICH PREMATURE LABOUR WAS INDUCED BY THE WATER DOUCHE.}

\author{
BY J. PRIOR LACY, EsQ., M.R.C.S.,
} SENIOR SURGEON TO THE NEWARK HOSPITAL.

L. C- - aged thirty-seven, a woman of middle stature, fair complexion, phlegmatic temperament, and good general health, is the wife of an agricultural labourer; she began to menstruate at seventeen, and continued to do so regularly till after her marriage, at the age of thirty-two. Has had no miscarriages, and the present is her fourth pregnancy. This woman, on account of congenital deformity of the pelvis, consisting of great projection of the promontory of the sacrum, narrowing of the pubic arch, and consequent lessening of the antero-posterior diameter, was, in her two first labours, which occurred at the full time, obliged to be delivered by means of craniotomy. The first child was a boy, the second a girl.

In her third pregnancy she was admitted into the Newark hospital, and premature lahour induced at seven and a half months, by puncturing the membranes; the presentation was an arm, the labour protrarted, considerable difficulty was experienced in turning, and the child, a girl, born dead.

On the occurrence of her fourth pregnancy it was again determined to admit her into the Newark hospital, and induce premature labour at seven and a half months, with a view of saving the life of the child. She became an in-patiert on October 29th, 1852, and the novel mode of cperation described and advocated by Dr. 'Tyler Smith, in The Lancet of Oetober 2nd, 1852, was pretty closuly followed, except that no syphon was employed, but in its stead the distal end of the india-rubber tube was fastened to a receptacle, placed ten feet above the patient, and the other end of the tube introduced into the vagina without any enema pip» being affxed to it. When the patient was Iying in bed the os uteri could with difficulty be felt high up and near the pubes; but on placing her in the bath, the alteration of position caused it to be felt much more readily. A dose of castor-oil was given on admission, which operated freely, and on-

October 30th-halt-past nine A.3r.-the patient being placed in an open bath, and the uterine end of the india-rubber tnbe introduced into the vagina, and held onposite the os uteri, two and a hal gallons of water, at $110^{\circ}$ Fahr., were poured into the receptacle; as soon as this had escaped through the tuhe the same quantity of cold water was poured in, and suffered to flow against the os uteri in like manner. The patient complained of some uneasiness when the cold current first began to ran.

Three $P$ ir.-The douche was repeated in the same manner as last described, but commencing with cold instead of warm water. Since the first douche, micturition has been rather frequent, but little pain has been experienced.

Nine P.M.. - The douche was repeated, commencing with wara water; the end of the forefinger can be introduced into the os nteri, but no pain of any consequence has occurred.
31st.-Half-past nine A.M : The patient has passed a good night, and feels comfortable; the bowels have been again relieved; micturition frequent; slight pain in the back and abdomen; douche repeated, commencing with warm water.-Halfpast two P.M. : Some slight shivering, nausea, and giddiness have been experienced since last report; douche repeated, commencing with two and a half gallons of cold, followed by five gallons of warm water. - Half-past eight P.M.: The symptoms continue pretty much as at last report; douche repeated in the usual manner and quantity, commencing with warm water. Though no perceptible change in the state of the os nteri has occurred, the patient whilst in the bath experienced increased shivering, nausea, giddiness, and pain in the back. Within an hour after the application of the douche, she had some rather severe rigors, and pains continued to increase in regularity and intensity till half-past eleven P.Mr. On making an examination at this time, the os was found as large as a crown-piece, the bag of membranes protruding, conical in shape, and very tense, even in the absence of pains.

Nov. 1st.-Half-past one A.M. : The os is fully dilated, and the bag of membranes has almost reached the os externum ; the presentation was found to be either hand or foot, but the membranes were too tense to determine which; consequently, as the pains continued regular and severe, it was decided to evacuate the liquor amnii, so that in the event of its heing necessary, turning might be immediately performed. On rupturing the membranes, an unusual quantity of water was discharged; one foot was found presenting, and being brought down, was secured by a noose. Pains now ceased almost entirely, and the patient obtained some sleep during the remainder of the night. At eleven A.M., she had an enema of warm water, which was repeated at two P.M. : Some slight pains succeeded. At half-past two p.Mr., the vagina was injected with warm water. No progress being made, a scruple of powdered ergot was given at three P.M., and repeated at half-past three and four. Labour pains of considerable intensity commenced soon after the first dose, and continued to increase till half-past four, at which time a still-born male child was expelled. The head was rather large and firmly ossified, but considerably moulded by its passage through the contracted brim. The placenta followed almost immediately. At bed-time the patient took an anodyne draught, followed by a dose of castor-oil in the morning. The bowels were comfortably relieved. The afterpains were slight, and the secretion of lochia and milk was namral. The recovery was rapid and uninterrupted.

Labour commenced immediately after the sixth use of the douche, and thinty-six hours after the first use of the douche. Duration of labour, eighteen hours.

The account of the foregoing case has been most correctly drawn up by Mr. Rake, the house-surgeon to the hospital. It is evident, that in this instance, though the child was lost, the chances of its being born alive were considerably greater than if the merabrane had been punctured before the commencement of labour. In the present case, the membranes were ruptured after the os uteri had been completely dilated by the influence of the douche. Should another case of this description come under my care, I should allow the liquor amnii to be discharged without any interference. The patient returned to her home in the country a fortnight after her delivery; and in passing through the village in which she resides a few days ayo, $Y$ called and found her quite well, and in the full employment of her domestic duties. I should wish to state, that I resurted to the operation entirely from perusing the successiul case related by Or. Tyler Smith in The Lincet of̈ October 2 nd.

Novembers 1852.

\section{ON THE TREATMENT OF ERYSIPELAS.}

\section{By VINCENT LITCHFIELD, Eso., M.R.C.S., Twickenham.}

IT is now some time since, that I began the treatment of erysipelas by means of the tinct. ferri sesquichlor.; and finding the results so satisfactory, I am induced to record a vote in its favour. I was led to make a trial of it at the recommendation of Dr. Brown, of Edinbugh, who kind!y sent me a pamphlet on the subject, written by Mr. Hamilton Bell. He says, "I have resorted to this mode of treatment for a quarter of a century, without having in a single instance failed of sucesss." This was boldly penned, and I, for one, did not thoroughly believe it; but I an too glad to state now, that I believe I could cure many cases in a week, which I formerly should have been a month at. Mr. Bell goes on to state the mode of administration, and expresses so much, and that too so aptly, in a few lines, that $I$ cannot do better than transcribe them. He says:-"Of' course the first object is to have the bowels freely acted on. If the erysipelas be mild, fifteen drops of the iron are administered in water every two 